\title{
Adolescents had poor knowledge about Papanicolaou (cervical) smear screening and identified many barriers to being screened
}

Kahn JA, Chiou V,Allen JD, et al. Beliefs about Papanicolaou smears and compliance with Papanicolaou smear follow-up in adolescents. Arch Pediatr Adolesc Med 1999 Oct;153:1046-54.

QUESTION: How do adolescent girls understand and perceive Papanicolaou (Pap or cervical) smear screening and barriers to compliance?

Design

Qualitative study using focus groups and indepth, semistructured interviews.

Setting

2 clinics in a children's hospital in Boston, Massachusetts, USA.

\section{Participants}

15 adolescents (mean age 18.7 y, 8 African-Americans, 5 with children, 11 in school, 7 with a history of sexually transmitted diseases) were approached and all had individual interviews. Adolescents who had been sexually active and who had normal and abnormal cervical smears were selected. Only a proportion of the latter group returned for follow up.

\section{Methods}

The interview guide was developed from data in 3 focus groups with 12 adolescents. The content was derived from 4 models (health belief model, social cognitive theory, theory of planned behaviour, and transtheoretical model and stages of change). The individual interview guide included questions and a scenario about health concerns; knowledge about pelvic examinations and cervical smears; perceived benefits, barriers, and susceptibility; information seeking; and suggestions for overcoming barriers to screening, increasing awareness of the importance of cervical smears, and improving appointment keeping.

\section{Main findings}

Girls reported that their peers were concerned about sexually transmitted diseases, pregnancy, breast cancer, abnormal cervical smears, cervical cancer, and smoking, but they themselves had little knowledge about cervical smears (11 had poor knowledge). 9 thought that their peers had cervical smears. Prevention and early detection of cervical cancer were benefits of the procedure. Perceived barriers were pain $(n=13)$, embarrassment $(n=10)$, fear of finding a problem or of the unknown $(\mathrm{n}=11)$, denial or perceived invincibility $(\mathrm{n}=6)$, health provider characteristics $(n=6)$, not seeking out trouble $(n=4)$, lack of knowledge $(n=4)$, fear of parents finding out, prolonged clinic waiting time, fear of finding out that they were pregnant, impulsiveness, cost, taking time off, child care, transportation, time, and energy. 7 girls reported high perceived susceptibility to cervical cancer. According to the participants, information about an abnormal smear could be obtained from physicians, clinics, gynaecologists, school nurses, libraries, family, friends, the internet, print material, counsellors, and telephone hot lines. Suggested methods to overcome barriers were education, development of a trusting, ongoing relationship with care providers who could put patients at ease and communicate well, gentle examinations, assured confidentiality, and school programmes. Compliance with appointment keeping could be enhanced with telephone or postal reminders, expanded hours, shorter waiting times, provision of babysitting or transportation, and ensuring that someone answered the phone when patients phoned to book appointments.

\section{Conclusions}

Adolescent knowledge about cervical smears was poor. They identified many barriers to being screened and ways to overcome barriers.

\section{COMMENTARY}

Adolescent girls' beliefs and attitudes about cervical screening are explored in the study by Kahn $e t$ al. Understanding adolescent girls' perspectives concerning cervical smears and overcoming barriers to compliance are fundamental to the prevention of cervical cancer and to public health promotion. Moreover, strategies presented by adolescent girls to enhance their compliance with cervical smear screening appointments are particularly relevant to clinical practice and health education.

The study also addressed the importance of education at the time of the cervical examination. However, helping adolescent girls to understand their cervical examination well in advance of the experience may provide an opportunity for developing positive attitudes and a well informed adolescent client. As Jorgenson points out, "sexual activity is being initiated at younger ages and with a greater average number of partners during the adolescent years"'; these factors may increase an adolescent's risk of cervical cancer. Additional research to evaluate the inclusion of health related information about the cervix when teaching about menstruation may be warranted.

The practice of educating adolescent peer helpers about cervical health, with opportunities for teaching younger students (age 12-13 y), may both educate and enhance self confidence in overcoming barriers to follow up care. The study raises the question, does earlier teaching promote greater awareness of cervical cancer risk reduction and responsible decision making in sexual health behaviour?

This study supports the evidence that individuals are more likely to seek health care when they feel comfortable with health and medical personnel. Lastly, in our work to educate and encourage responsible decision making for health, we can recognise and adopt the study methods that promote proactive behaviour in clients and a more adolescent friendly healthcare system.

Maureen Hobbs, RN, BScN Public Health Nurse Capital Health Region Community Health Services Victoria, British Columbia, Canada

1 Jorgenson SR. Adolescent pregnancy and parenting. In Thomas G, Adams G, Montemayer R, editors. Adolescent sexuality. California: Sage Publications, 1993:103-37.
Cincinnati, $\mathrm{OH} 45229$,

USA. Fax +1 513636

8844 . 